\title{
HADAMARD PRODUCTS OF SCHLICHT FUNCTIONS
}

\author{
LAWRENCE ZALCMAN ${ }^{1}$
}

1. Introduction. Let $D=\{z|| z \mid<1\}$ be the open unit disc and let $A(D)$ be the set of all functions analytic in $D$. A function $f \in A(D)$ is said to have valence $p$ in $D$ ( $p$ a natural number or $\infty$ ) if the equation

$$
f(z)=x
$$

has, for each fixed $x$, no more than $p$ distinct roots and if, for some value $x=x_{0}$, (1) has exactly $p$ distinct roots. Denote by $V_{D}(1)$ the subset of $A(D)$ consisting of the schlicht functions (i.e., the functions of valence 1 ) of the form

$$
f(z)=z+\sum_{n=2}^{\infty} a_{n} z^{n}
$$

Recently, A. W. Goodman showed that the sum or product of two functions in $V_{D}(1)$ may have valence $\infty$ [2]. In this paper, we are concerned with the behavior of $V_{D}(1)$ under a different composition, the familiar Hadamard product

$$
\left(\sum_{n=1}^{\infty} a_{n} z^{n}, \sum_{n=1}^{\infty} b_{n} z^{n}\right) \stackrel{*}{\rightarrow} \sum_{n=1}^{\infty} a_{n} b_{n} z^{n}, \quad a_{1}=b_{1}=1 .
$$

Our principal result is that the Hadamard product of two functions in $V_{D}(1)$ may have valence $\infty$; moreover, the functions involved may be taken to satisfy certain fairly strong "regularity" requirements (details are in $\$ 2$ ). In $\$ 3$, we state some complementary results due to Robertson. Finally, in $\$ 4$ we mention a related, apparently more difficult, problem.

2. The main example. Let $\epsilon>0$ be given. We shall construct a function

$$
h(z)=z+\sum_{n=2}^{\infty} c_{n} z^{n}
$$

such that

(i) the $c_{n}$ are real,

(ii) $\sum_{n=2}^{\infty}\left|c_{n}\right| \leqq 1 / 2+\epsilon$,

Received by the editors March 4, 1967.

1 National Science Foundation Graduate Fellow. 
(iii) $h(z)$ has infinite valence.

Suppose this construction has been accomplished and that $\epsilon \leqq 1 / 2$. Let

$$
\begin{aligned}
& f(z)=\frac{z}{(1-z)^{2}}=z+\sum_{n=2}^{\infty} n z^{n} \\
& g(z)=z+\sum_{n=2}^{\infty} \frac{c_{n}}{n} z^{n} .
\end{aligned}
$$

Clearly, $f$ is schlicht, starlike, and typically real. Since $\sum_{n=2}^{\infty} n\left|c_{n} / n\right|$ $=\sum_{n=2}^{\infty}\left|c_{n}\right| \leqq 1, g$ is also schlicht and starlike (see, for instance, [5]); furthermore, $g$ is typically real since the $c_{n}$ are real. Finally, $(f * g)(z)=h(z)$, a function of infinite valence. It remains to construct $h$ as required.

Let $\left\{x_{n}\right\}$ be a sequence of real numbers, $0<x_{n}<1$, such that $\prod_{n=1}^{\infty} x_{n}$ does not diverge to 0 . Let

$$
B(z)=\prod_{n=1}^{\infty} \frac{x_{n}-z}{1-x_{n} z}
$$

be the Blaschke product associated with $\left\{x_{n}\right\} . B$ is analytic on $D$ and satisfies $|B(z)|<1$ there. Also,

$$
B^{\prime}(z)=\sum_{n=1}^{\infty}\left\{\frac{x_{n}^{2}-1}{\left(1-x_{n} z\right)^{2}} \prod_{j \neq n} \frac{x_{j}-z}{1-x_{j} z}\right\}
$$

so that

$$
\left|B^{\prime}(z)\right| \leqq 4 \sum_{n=1}^{\infty} \frac{1-x_{n}^{2}}{|1-z|^{2}} .
$$

Given $\delta>0$,"we can choose the $\left\{x_{n}\right\}$ so that

$$
\begin{aligned}
1-\delta & <\prod_{n=1}^{\infty} x_{n}=B(0), \\
\left|(z-1)^{2} B^{\prime}(z)\right| & \leqq 4 \sum_{n=1}^{\infty}\left(1-x_{n}^{2}\right)<\delta, \quad z \in D, \\
\left|B^{\prime \prime}(0)\right| & <\delta .
\end{aligned}
$$

Let $F(z)=(z-1)^{2} B(z)$. Then

$$
\begin{aligned}
\left|F^{\prime}(z)\right| & =\left|2(z-1) B(z)+(z-1)^{2} B^{\prime}(z)\right| \\
& \leqq 2|(z-1) B(z)|+\left|(z-1)^{2} B^{\prime}(z)\right|<4+\delta
\end{aligned}
$$


for $z \in D$. Thus $F^{\prime}(z)$ is a bounded analytic function on $D$. Let

$$
F^{\prime}(z)=\sum_{n=0}^{\infty} f_{n} z^{n}
$$

We have

$$
\left(\sum_{n=0}^{\infty}\left|f_{n}\right|^{2}\right)^{1 / 2}=\lim _{r \rightarrow 1}\left(\frac{1}{2 \pi} \int_{0}^{2 \pi}\left|F^{\prime}\left(r e^{i \theta}\right)\right|^{2} d \theta\right)^{1 / 2}<4+\delta .
$$

Let

$$
\begin{aligned}
h(z) & =\frac{F(z)-F(0)}{F^{\prime}(0)}=z+\frac{f_{1}}{2 f_{0}} z^{2}+\cdots \\
& =\frac{1}{f_{0}} \sum_{n=0}^{\infty} \frac{f_{n}}{n+1} z^{n+1} .
\end{aligned}
$$

Clearly $h(z)$ has infinite valence in $D$; indeed,

$$
h\left(x_{n}\right)=-F(0) / F^{\prime}(0), \quad n=1,2, \cdots .
$$

Now

$$
f_{0}=-2 B(0)+B^{\prime}(0), \quad f_{1}=2 B(0)-4 B^{\prime}(0)+B^{\prime \prime}(0)
$$

and the expressions for the $f_{n}, n \geqq 2$, involve $B^{\prime}(0), B^{\prime \prime}(0), \cdots$ but not $B(0)$. Thus for fixed $N$ we can arrange that

$$
\sum_{n=2}^{N-1}\left|\frac{f_{n}}{n+1}\right|<\delta
$$

by an appropriate choice of the $\left\{x_{n}\right\}$ (consistent with (9)). Choose $N$ such that

$$
\left(\sum_{n=N+1}^{\infty} \frac{1}{n^{2}}\right)^{1 / 2}<\delta
$$

Then

$$
\sum_{n=N}^{\infty}\left|\frac{f_{n}}{n+1}\right| \leqq\left(\sum_{n=N}^{\infty}\left|f_{n}\right|^{2}\right)^{1 / 2}\left(\sum_{n=N+1}^{\infty} \frac{1}{n^{2}}\right)^{1 / 2}<(4+\delta) \delta .
$$

From (9) and (15) we have

$$
\begin{aligned}
& \left|f_{0}\right|>2|B(0)|-\left|B^{\prime}(0)\right|>2-3 \delta \\
& \left|f_{1}\right|<2|B(0)|+4\left|B^{\prime}(0)\right|+\left|B^{\prime \prime}(0)\right|<2+6 \hat{o} .
\end{aligned}
$$

Thus 


$$
\begin{aligned}
\frac{1}{\left|f_{0}\right|} \sum_{n=1}^{\infty}\left|\frac{f_{n}}{n+1}\right| & =\frac{1}{\left|f_{0}\right|}\left\{\frac{\left|f_{1}\right|}{2}+\sum_{n=2}^{N-1} \frac{\left|f_{n}\right|}{n+1}+\sum_{n=N}^{\infty} \frac{\left|f_{n}\right|}{n+1}\right\} \\
& <\frac{1}{2-3 \delta}\left\{1+8 \delta+\delta^{2}\right\} .
\end{aligned}
$$

Choosing $\delta$ so small that $\left(19 \delta+2 \delta^{2}\right) /(4-6 \delta)<\epsilon$, we obtain

$$
\sum_{n=2}^{\infty}\left|c_{n}\right|=\sum_{n=1}^{\infty} \frac{1}{\left|f_{0}\right|} \frac{\left|f_{n}\right|}{n+1}<\frac{1}{2}+\epsilon .
$$

It is easy to see that we can choose $g(z)$ to be starlike at least of order $\alpha=(2 / 3)-\eta$, where $\eta>0$ can be made arbitrarily small. Indeed, by a result in [5], it is enough to show

$$
\sum_{n=2}^{\infty}(n-\alpha) \frac{\left|c_{n}\right|}{n} \leqq 1-\alpha .
$$

Choose $h(z)=z+\sum_{n=2}^{\infty} c_{n} z^{n}$ as above with $\left|c_{2}\right|>\frac{1}{2}+\epsilon / 2$ and $\sum_{n=2}^{\infty}\left|c_{n}\right|$ $<\frac{1}{2}+\epsilon$, where $10 \epsilon /(9-3 \epsilon)<\eta$. An easy computation then shows that (22) holds.

We can collect the results of this section into

THEOREM 1. Let $\eta>0$. There exist functions $f, g \in V_{D}(1)$, starlike and typically real, such that $f * g$ has infinite valence in $D . g$ may be taken to have an absolutely convergent Taylor series and to be starlike at least of order $(2 / 3)-\eta$.

3. Further results. Some positive results due to Robertson [6], [7] lend perspective to the example in $\$ 2$. We state them without proof.

THEOREM 2. Let $f, g \in V_{D}(1)$ be typically real and convex in the direction of the imaginary axis. Then $f * g \in V_{D}(1)$, and $f * g$ is typically real and convex in the direction of the imaginary axis.

TheOREM 3. Let

$$
f(z)=\frac{1}{z}+\sum_{n=1}^{\infty} a_{n} z^{n}, \quad g(z)=\frac{1}{z}+\sum_{n=1}^{\infty} b_{n} z^{n}
$$

be regular and schlicht in $0<|z|<1$. Then

$$
h(z)=\frac{1}{z}+\sum_{n=1}^{\infty} a_{n} b_{n} z^{n}
$$

is regular and schlicht in $0<|z|<1$. 
4. A related problem. Before closing, we should mention a related problem. Define a new composition on pairs of functions in $V_{D}(1)$ by

$$
\left(\sum_{n=1}^{\infty} a_{n} z^{n}, \sum_{n=1}^{\infty} b_{n} z^{n}\right) \stackrel{\otimes}{\longrightarrow} \sum_{n=1}^{\infty} \frac{a_{n} b_{n}}{n} z^{n} .
$$

Robertson [6] showed that if $f$ and $g\left(\in V_{D}(1)\right)$ are typically real then so is $f \otimes g$. On the other hand, $V_{D}(1)$ is not closed under $\otimes$; proofs of this fact are in [1], [3], and [4]. If $f, g \in V_{D}(1)$, can $f \otimes g$ have infinite valence in $D$ ?

\section{REFERENCES}

1. B. Epstein and I. J. Schoenberg, On a conjecture concerning schlicht functions, Bull. Amer. Math. Soc. 65 (1959), 273-275.

2. A. W. Goodman, The valence of sums and products, Canad. J. Math. (to appear).

3. W. K. Hayman, On the coefficients of univalent functions, Proc. Cambridge Philos. Soc. 55 (1959), 373-374.

4. C. Loewner and E. Netanyahu, On some compositions of Hadamard type in classes of analytic functions, Bull. Amer. Math. Soc. 65 (1959), 284-286.

5. E. P. Merkes, M. S. Robertson and W. T. Scott, On products of starlike functions, Proc. Amer. Math. Soc. 13 (1962), 960-964.

6. M. S. Robertson, Applications of a lemma of Fejer to typically real functions, Proc. Amer. Math. Soc. 1 (1950), 555-561

7. M. S. Robertson, Convolutions of schlicht functions, Proc. Amer. Math. Soc. 13 (1962), 585-589.

Massachusetts Institute of Technology 